3000 kilometres from the photosphere without producing an amount of pressure at its base which certainly does not exist. Yet the spectroscope tells us that incandescent hydrogen is actually present at a couple of hundred times that height. Nor can the anomaly be reconciled by supposing, with our author, the solar atmosphere to be chiefly composed of a very much lighter gas (" r 474 "), merely, as it were, adulterated with hydrogen. Even if we were satisfied to ascribe to "coronium" an almost impossible degree of elasticity, it would not avail to lift the mingled hydrogen one inch above its natural level. The law of diffusion does not abrogate the law of gravity. Each gaseous ingredient of a mixed atmosphere obeys its own law of equilibrium, as if it existed alone. It is true that the anticipated thinning out of oxygen at great heights in our own atmosphere has not been experimentally verified; but the incessant agitation of the air is believed to mask an effect which should otherwise be perceptible.

The action of a repulsive force, such as is visibly exerted on comets, has been invoked as a means of escape from this difficulty. The supposition has much to recommend it, and would remove a good deal of perplexity; it is, besides, countenanced by the authority of Dr Huggins. But the more tempting it appears, the more severely it should be tested previous to its admission, on other than a provisional footing, among the theories of science.

The slightness of resistance to motion in the solar neighbourhood is one among many indications of the extreme tenuity of matter there. Comets well-nigh graze the sun's surface without experiencing perceptible retardation; and millions of cubic miles of hydrogen sweep onwards or upwards at rates up to 250 miles a second, almost as if in vacuo. Since both the moving substance and the medium are incandescent, the varying viscosity of gases at high temperatures would claim attentive consideration in the matter, were it not that reliable data are unfortunately deficient.

The rotational peculiarity of the sun may, however, be said to dominate the problem of its constitution. Three classes of explanation are possible, and have found various degrees of favour. It may be produced by the fall of matter upon the photosphere, by the ascent of matter from beneath it, or by surface-currents. The last was the theory of Zöllner, and has been inherited from him by $M$. Schulz, but may be dismissed without hesitation as contradictory of known facts. M. Faye's hypothesis of vertical currents bringing up with them a smaller linear velocity is more plausible, but needs peremptory treatment to fit it to the required shape. It is remarkable that M. Belopolsky has lately deduced theoretically, on some not improbable assumptions, Spörer's empirical formula of the diminishing rate of the sun's rotation north and south from his equator (see NATURE, vol. xxxiv. p. 54). The coincidence is striking; but it must not mislead us. It is not enough that a cause be true; it must also be sufficient. Is there any likelihood of its being such in this case? We apprehend that the effects, even supposing them realised to the full, would be microscopic compared with those actually observed. If we take the mean density of the sun at double its superficial density (an outside admission), the maximum of gravity will occur below the surface, at a depth of one-sixth of the radius, and there the theoretical rotation-period comes out, by a rough calculation, about twenty-two days. But this period is not in reality complied with. The tremendous hindering power of friction intervenes. It occurs on paper only, and belongs even there but to a single stratum. The effects in superficial acceleration must be quite inconsiderable.

Acceleration from below failing, we turn to acceleration from above. And it has to be borne in mind that the mode of the sun's rotation is inferred from the movements of spots, and from them alone. But if spots be due, as generally supposed, to vaporous down-rushes, they must share in the augmented velocity brought by the materials forming them from regions of wider circumference; and this theoretical necessity is confirmed by the characteristic plunge forward attending the sudden development of these objects. If we assume further that the height of fall, consequently the added linear velocity, diminishes progressively with distance from the equator, the phenomena of spot-transport in longitude are satisfactorily accounted for. Just such a graduated elevation of the sources of spot-supply forms an integral part of Mr. Lockyer's "meteoric-ring theory" of sunspots, communicated to the Royal Society in May last (NATURE, vol. xxxiv. p. 25I). The line thus struck out, however it may be modified by future experience, seems to lead, more naturally and easily than any other yet tried, to the solution of the problem of the sun's apparent rotation.

A. M. CleRKE

\section{THE NEW OPTICAL GLASS}

FVERYTHING that falls from the pen of Prof. Abbé of Jena relating to optical matters commands respect. His announcement therefore of the discovery of new kinds of glass specially adapted for the manufacture of lenses has been received with peculiar interest; and although details of information on various points are still wanting, enough has been published respecting the new optical glass to be worthy of more than passing notice.

All who have had anything to do with optical instrument-making know only too well the existence of the defect termed "irrationality of dispersion." When Hall and Dollond had independently shown that the chromatic dispersion of a crown glass lens might be corrected by combining it with a second lens of flint glass, a new impetus was given to optical research. The so-called "achromatic" lens in the hands of successive generations of opticians constituted the basis both of the modern microscope and of the modern telescope. But as greater and greater perfection in the construction of the "achromatic" lens was attained, it became apparent that perfect achromaticity was very far from being realised; for though two lenses might be found which should perfectly bring together two widely differing rays, such, for example, as the red of the line $C$ of the spectrum and the blue of line $G$ of the spectrum, it by no means followed that this pair of lenses would bring together to the same focus all other rays. On the contrary, owing to the "irrationality" of dispersion a "secondary spectrum" would always remain uncorrected.

The relation between the chemical constitution of a medium and its action on waves of light of different periods is one of those matters about which we are still profoundly ignorant. We know that a prism of glass does not spread out the waves in proportion to their wave-length, or to the frequency of their periods. A simple case of irrationality is afforded in the fact that a green ray which when viewed through one prism may lie exactly half-way between $C$ and $G$ in the spectrum will not lie exactly half-way when viewed through a prism of a different kind of glass. All that a combination of two lenses can do is to achromatise for two rays of the spectrum: it may very nearly achromatise for the neighbouring rays, but strictly speaking it only achromatises for two. For ordinary optical purposes we seek to achromatise for the red and the blue, so reconciling the end regions of the visible spectrum. For photographic purposes we achromatise for green and violet (or even ultra-violet) rays, reconciling the end regions of the photographically active spectrum.

To Dr. Blair, whose observations were published in the Transactions of the Royal Society of Edinburgh for I791, we owe the suggestion to achromatise for three rays by using compound lenses of three different media. Blair, indeed made a most extensive examination into the dispersive powers of various media, and in particular of 
liquid media, which he proposed to use in his compound lenses ; a fluid lens being formed in a cavity between two lenses of glass. He also suggested combinations of two or more fluid lenses. He found that in the spectrum of hydrochloric acid the green rays lay much nearer the violet than in the spectra of most metallic solutions; and he proposed to use the chlorides of antimony and of mercury in various proportions along with hydrochloric acid, or with sal-ammoniac, in order to obtain a fluid which, while having a different absolute dispersion from crown glass, should have its relative dispersion or relative distribution of the rays of different colours proportionately identical throughout the spectrum. Blair's object-glasse for telescopes, though regarded at the time as of singular merit, never came into use. The only recent serious attempt to revive a triple lens for approximating to a correction of the secondary spectrum by achromatising for three rays has been that of Prof. C. S. Hastings, of Yale, who has used three kinds of glass.

What Blair proposed to effect with his liquid combinations Prof. Abbé claims to have now achieved by his discovery of new kinds of optical glass. To Abbé we owe the remark that, in addition to the secondary chromatic aberration of our so-called achromatic lenses, there exists a second defect, termed by him the chromatic difference of the spherical aberrations. 'This term he uses to denote the fact that with the crown and flint glasses used by opticians, though the curves of the lenses be calculated to correct the spherical aberration, taking in each case the mean refractive power, there will be a slight residual spherical aberration for all rays not of mean refractive index; the lens being spherically under-corrected for red rays and spherically over-corrected for blue.

Having realised so far back as 1880 that these defects were inherent in the use of such glass as opticians had at their disposal, Abbé determined to make a resolute attempt to discover new kinds of glass which should be free from these vices. The research involved no less a field of work than the examination of the optical properties of all known chemical substances which undergo vitreous fusion and solidify in non-crystalline transparent masses, together with a detailed comparison of their physical and chemical properties. The work was begun so far back as January of the year I88I by Prof. Abbé and his coadjutor, Dr. Schott, then of Witten in Westphalia, now of Jena. Dr. Schott undertook the chemical portion and the melting processes ; Prof. Abbé and his assistant, Dr. Riedel, conducted the optical examinations of the products. At first only small quantities, from 20 to 60 grammes in weight, were melted at once ; all kinds of chemical elements being tried with the view of ascertaining their influence on the refractive and dispersive powers. Not only were glasses of ordinary kinds having silicic acid for their chief constituent tried, but also glasses, resembling Faraday's famous "heavy-glass," made from boric acid and also phosphoric acid. So, by the end of I $88 \mathrm{I}$, a series of fundamental facts in chemical optics were gathered together for future use. The next point was to use these chemical researches as the basis for the production of real glass possessing the necessary qualities of hardness, colourlessness, and unalterability. To carry out this work, Dr. Schott moved to Jena early in I 882, and set up a special laboratory for scientific glassmaking with proper blast-furnaces, and smelting-pots in which quantities of Io kilogrammes could be melted at once. Until the end of the year 1883 this researchlaboratory was occupied almost exclusively in working toward the solution of two practical problems. The first of these was the production of pairs of kinds of flint and crown glass, such that the dispersion in the various regions of the spectrum should be, for each pair, as nearly as possible proportional. The object of this was to permit of a higher degree of achromaticity than hitherto possible, by diminishing the secondary colouring effects inseparable from the irrationality of the ordinary silicate flint and crown glasies. The second problem was the production of a greater multiplicity in the gradations of optical glass in respect of the two chief optical constants, the index of refraction and the mean dispersion. Though this has not generally been regarded as an important need in optics, it was considered by Prof. Abbé and Dr. Schott as of quite equal importance to the first. For the silica glasses hitherto used constitute, according to their composition, a simple series, from the lightest crown to the densest flint, in which, with an increase in refractive index, there is practically always an increase in the mean dispersion. But there is no doubt that for many purposes of practical optical instrument-making, particularly in the designing of optical combinations for special purposes, it would be a great benefit for the optician to have at his command other kinds of glass in which the refraction and dispersion are not related in the way in which they are in the silica glasses ; for example, a glass having great refraction and small dispersion. Hence the multiplication in the available kinds of optical glass opens out new possibilities of great practical moment. Pending the publication of these very valuable scientific investigations, only a very brief account can be given of the actual results. The first problem has been satisfactorily solved, with the result that achromatic lenses of a much more perfect kind than has ever been attainable are now in the market; and the second has also been successfully carried out, a whole series of new glasses of graduated properties having been introduced into the optical trade.

Down to the autumn of 1883 the matter was one of systematic scientific research; but at that date, encouraged by the substantial fruit borne by the investigations, a further step was taken. In conjunction with the brothers Dr. Carl Zeiss and Dr. Rod. Zeiss, of Jena, whose names are household words to every microscopist, a commercial enterprise was set on foot for establishing a new glassfoundry. This establishment, which, under the style of Schott and Company, has been at work since the autumn of 1884 , produces glass of all kinds, old and new, on a large scale. The first application of the new glass to the microscope comes naturally from the famous firm of Zeiss. To his new microscope lenses Prof. Abbé gives the distinctive name of apochromatic objectives. $\mathrm{He}$ claims for them great superiority in the finer qualities of definition, the new dry apochromatic lens giving an image equal to that of an ordinary achromatic water-immersion objective. He also claims that the more perfect corrections permit equal magnification to be obtained by using a longer-focus objective with an eye-piece of higher power than hitherto has been usual, thus obviating some of the difficulties of very short-focus objectives. Moreover the foci for visible and for photographic purposes are identical. Special compensating eye-pieces have been also devised for use with the new apochromatic objectives.

Whether these new appiiances are found to fulfil under the test of experience all that their inventor claims for them remains yet to be seen ; but it cannot be doubted that a great step has been taken. It ought also to be recorded to the credit of all concerned that no attempt is being made to secure to one firm a monopoly of the new materials, but that the new optical glass is offered to the optical trade without any restriction or patent to stand in the way of further development. Nor less honourable or significant is it that the researches of the "Glass-technical Laboratory" of Jena should have been supported by a very liberal and several times repeated subvention from the Prussian Government. Such a result arising from the endowment of research makes ample answer to the easily. uttered assertion that such endowments, if given, would be wasted on useless fancies and trifling schemes. We sincerely congratulate Prof. Abbe and Dr. Schott on the completion of their most meritorious labours. 